\title{
Spinal cord stimulation may improve not only intractable pain but also necrotic wounds
}

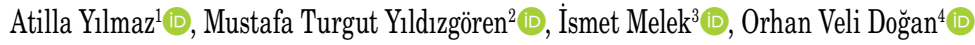 \\ ${ }^{1}$ Department of Neurosurgery, Mustafa Kemal University Medical School, Hatay, Turkey \\ ${ }^{2}$ Department of Physical Medicine and Rehabilitation, Mustafa Kemal University Medical School, Hatay, Turkey \\ ${ }^{3}$ Department of Neurology, Mustafa Kemal University Medical School, Hatay, Turkey \\ ${ }^{4}$ Department of Cardiovascular Surgery, Mustafa Kemal University Medical School, Hatay, Turkey
}

Received: September 11, 2017 Accepted: October 26, 2017 Published online: February 22, 2018

\begin{abstract}
Spinal cord stimulation (SCS), an implantable neuromodulation modality, is one of the most exciting developments in chronic pain syndromes. In addition, SCS may improve intractable pain and may help ischemic wound healing. Herein, we report a 59-year-old female patient with persistent neuropathic pain and peripheral arterial disease in the lower limb which was treated successfully with SCS.
\end{abstract}

Keywords: Neuropathic pain; spinal cord stimulation; wound.

Spinal cord stimulation (SCS), an implantable neuromodulation modality, is one of the most exciting developments in chronic pain syndromes. It was originally based on the gate-control theory of pain proposed by Melzack and Wall; ${ }^{[1,2]}$ however, the research has shown that electrical stimulation of the spinal cord can result in further more complex pathophysiological changes. With the application of SCS, a local alteration is produced in wide-dynamicrange neuron excitability, physiological inhibitory mechanisms are facilitated, and there are changes in the activity of several neurotransmitters, primarily gamma-aminobutyric acid (GABA), but also glutamate, adenosine, acetylcholine, substance $\mathrm{P}$, calcitonin-gene related peptide (CGRP), brain-derived neurotrophic factor (BDNF), bradykinin, and others. ${ }^{[2,3]}$ Spinal cord stimulation is indicated in a number of clinical situations such as failed back surgery syndrome, degenerative low back pain, peripheral neuropathy, chronic regional pain syndromes type 1, postherpetic neuralgia, cancer pain, phantom pain, urge incontinence, refractory angina pectoris, and peripheral vascular pain. ${ }^{[4,5]}$ To treat lower limb pain, 4 to 8 electrodes are often placed in the epidural space between the thoracic
8 and 11 level through a paralaminar level 1 or 2 through lumbar needle or via mini laminectomy. ${ }^{[6]}$ The electrodes are usually centered in the spinal canal or directed off-center, depending on the location of the limb with the most significant symptoms. Herein, we report a case with persistent ischemic pain and necrotic wounds successfully treated with SCS implantation for inoperable peripheral vascular disease (PVD).

\section{CASE REPORT}

A 59-year-old female patient was admitted to our outpatient clinic due to persistent neuropathic pain in the lower limb, which started approximately two years ago. The pain score at rest reported on a 10-point Visual Analog Scale (VAS) was 8. Her past medical history revealed that she was diagnosed with diabetes mellitus and peripheral arterial disease, and spinal stenosis surgery was performed a year ago. On physical examination, the pulses were diminished on the right side, and the left leg was amputated above the knee. The patient had no prosthetic device, and used a wheelchair for mobilization. Necrotic wounds varying in size from $1 \times 1 \mathrm{~cm}$ to $1 \times 3 \mathrm{~cm}$ were seen on the right foot, which 

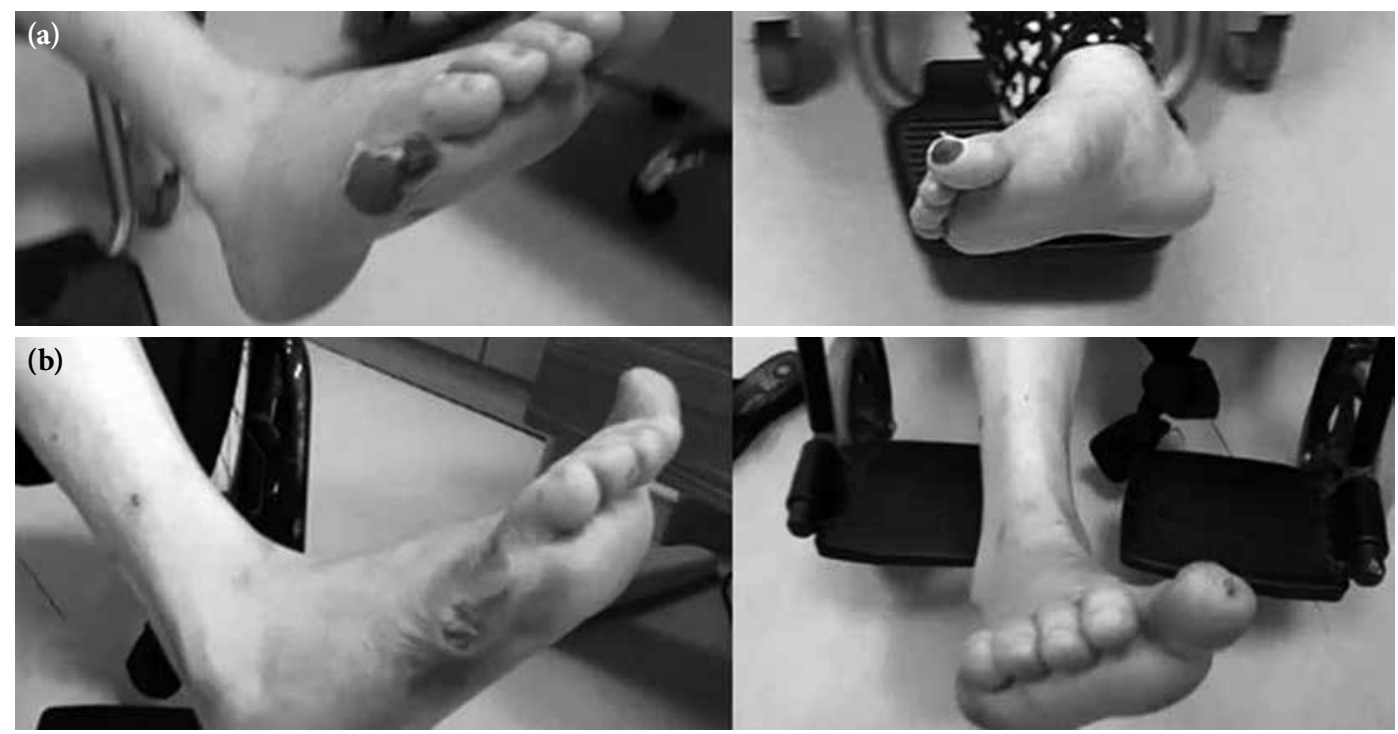

Figure 1. An image showing the necrotic wound (a) before and (b) after spinal cord stimulation treatment.

were present for six months (Figure 1a). She was treated with regular skin cleanings, dressing with antiseptics and topical antibiotics. The Fontaine stage was IV (rest pain and arterial ulcers) and the Ankle Brachial Index (ABI) was 0.60 on Doppler ultrasonography. Severe motor and sensory axonal neuropathy was observed on electroneuromyography. The right posterior tibial artery was totally occluded with distal reconstruction via collaterals to the ankle level according to angiography. Catheter embolectomy and balloon angioplasty were performed for limb ischemia. Recanalization was not achieved at the end of the angioplasty.

The patient was initially treated with vasodilators in addition to antiplatelet drugs. Naproxen sodium $(750 \mathrm{mg} / \mathrm{d})$ and gabapentin $(300 \mathrm{mg} / \mathrm{d})$ were also started with gabapentin progressively increased to $2400 \mathrm{mg} / \mathrm{d}$ and the subsequent addition of duloxetine $(60 \mathrm{mg} / \mathrm{d})$. The patient was clinically re-evaluated six weeks after angioplasty. The pain intensity was slightly decreased (VAS=7). At three months, duloxetine was discontinued and tramadol $(200 \mathrm{mg} / \mathrm{d})$ was added. However, treatment did not ameliorate the pain. At five months after treatment, she complained of numbness, coldness, and persistent pain at rest $(\mathrm{VAS}=8)$ in the lower limb. After administering these treatments, SCS trial was recommended. A 16-electrode array SCS device (Medtronic Inc., Minneapolis, MN, USA) was implanted via mini laminectomy due to previous spinal stenosis surgery due to severe pain and paresthesia (Figure 2). The leads were placed in the lower part of the $\mathrm{T}_{9-10}$ vertebral body. After successful trial stimulation, permanent SCS implantation was performed and the pain immediately decreased from VAS 8 to 3 . After one month of follow-up, in addition to adequate pain relief, a subjective improvement in peripheral coldness, wound healing, and limb mobility was observed (Figure 1b). The patient was satisfied with the SCS treatment and was only taking gabapentin at $1800 \mathrm{mg} / \mathrm{d}$.

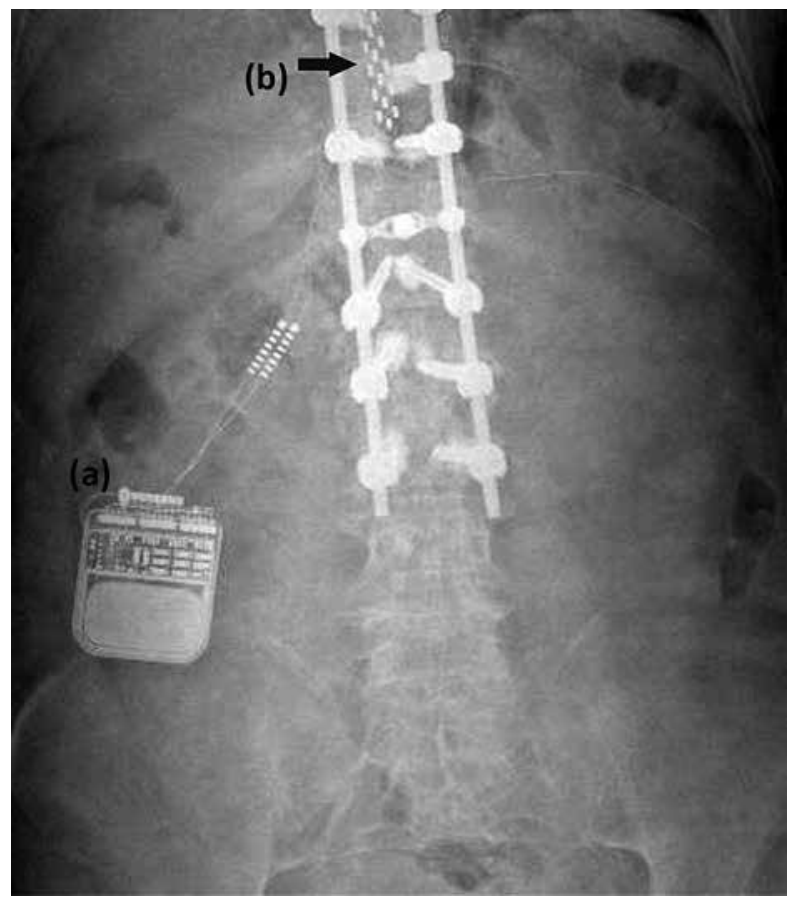

Figure 2. A permanent spinal cord stimulation device: (a) Anteroposterior radiograph showing the location of the spinal cord stimulator device and, (b) paddle leads (arrow). 


\section{DISCUSSION}

In 2013, the Neuromodulation Appropriateness Consensus Committee (NACC) presented the first comprehensive recommendations for the use of SCS for the treatment of chronic and ischemic pain. ${ }^{[7]}$ Based on the recommendations of the NACC, in PVD, compared to conservative treatment (i.e., analgesics, vasodilators, and anticoagulants), SCS may decrease amputation rates and improve pain in selected patients who are refractory to conservative medical treatment and reconstructive surgical procedures. ${ }^{[7]}$

Patients with chronic limb ischemia who are ineligible for open surgical or endovascular interventions (Fontaine stage III-IV) have been identified as candidates for SCS therapy, when they are not responsive to medical therapy. Although, the SCS mechanism is not completely understood yet, there are several theories about the pain relief effect. ${ }^{[8]}$ The effect may be related to the inhibition of the sympathetic nervous system through the stimulation and can, therefore, provide antidromic vasodilation, which reduces oxygen demand and increases blood flow. ${ }^{[8,9]}$ In addition, SCS may improve ischemic wound healing and offer benefit for limb preservation through improved skin perfusion.

In conclusion, our case, there was a history of concomitant peripheral artery disease and surgery for lumbar spinal stenosis and the patient was determined as Fontaine Stage IV and ABI 0.60. With this case report, we highlight the fact that SCS may improve intractable pain and may help ischemic wound healing, and can prevent or postpone limb amputation. Therefore, relevant patients should be followed rigorously to ensure prompt treatment. We suggest that providing pain relief with SCS may improve wound healing and mobilization.

\section{Declaration of conflicting interests}

The authors declared no conflicts of interest with respect to the authorship and/or publication of this article.

\section{Funding}

The authors received no financial support for the research and/or authorship of this article.

\section{REFERENCES}

1. Melzack R, Wall PD. Pain mechanisms: a new theory. Science 1965;150:971-9.

2. Foreman RD, Linderoth B. Neural mechanisms of spinal cord stimulation. Int Rev Neurobiol 2012;107:87-119.

3. Linderoth B, Meyerson B. Spinal cord stimulation: mechanisms of action. In: Burchiel K, editor. Surgical Management of Pain. 2nd ed. New York: Thieme Verlag; 2014. p. 319-33.

4. Cameron T. Safety and efficacy of spinal cord stimulation for the treatment of chronic pain: a 20-year literature review. J Neurosurg 2004;100:254-67.

5. Özdemir İ, Akbaş M1, Yeğin A, Dağıstan G, Erkan DÖ. Spinal cord stimulation in 62 patients: Retrospective evaluation. [Article in Turkish] Agri 2017;29:25-32.

6. Zhu J, Gutman G, Collins JG, Colonna J. A review of surgical techniques in spinal cord stimulator implantation to decrease the post-operative infection rate. J Spine 2014;4:202.

7. Deer TR, Mekhail N, Provenzano D, Pope J, Krames E, Leong $\mathrm{M}$, et al. The appropriate use of neurostimulation of the spinal cord and peripheral nervous system for the treatment of chronic pain and ischemic diseases: the Neuromodulation Appropriateness Consensus Committee. Neuromodulation 2014;17:515-50.

8. De Caridi G, Massara M, David A, Giardina M, La Spada M, Stilo F, et al. Spinal cord stimulation to achieve wound healing in a primary lower limb critical ischaemia referral centre. Int Wound J 2016;13:220-5.

9. De Caridi G, Massara M, Benedetto F, Tripodi P, Spinelli F, David A, et al. Adjuvant spinal cord stimulation improves wound healing of peripheral tissue loss due to steal syndrome of the hand: clinical challenge treating a difficult case. Int Wound J 2016;13:72-6. 